\title{
Facilitating and understanding the family's choice of injection device for growth hormone therapy by using conjoint analysis
}

\author{
S F Ahmed, ${ }^{1}$ W A Smith, ${ }^{1}$ C Blamires ${ }^{2}$
}

${ }^{1}$ Royal Hospital for Sick

Children, Yorkhill, Glasgow, UK;

${ }^{2}$ Decision Modelling

Consultancy Ltd, Lymm,

Cheshire, UK

Correspondence to:

Dr S Faisal Ahmed, Department

of Child Health, Royal Hospital

for Sick Children, Yorkhill,

Glasgow G3 8SJ, UK; s.f.

ahmed@clinmed.gla.ac.uk

Accepted 25 March 2007

Published Online First 5 April 2007

\section{ABSTRACT}

Background: Conjoint analysis involves the measurement of consumer preferences between choice alternatives.

Aims and objectives: To investigate the use of conjoint analysis in facilitating and understanding choice of growth hormone injection devices.

Method and subjects: 56 patients and their parents participated in an electronic, computer-based interview. The interview took a median time of 18 min (range 1230) and allowed an immediate matching of injection devices to the family's preferences.

Results: Amongst the key drivers of choice, lack of bruising was rated highest and designated an index of 100. Compared to this, the remaining attributes in order of desirability were: auto-injector (98), lack of pain (93), lightweight (88), silent (82), ready-mixed (77), ease of holding (69), telephone helpline (66), needle-free (62), small size (60), nurse support (47), hidden needle (45), stored in fridge (13) and home delivery (6). Out of the 17 families who had already chosen a device previously by discussion with the clinic nurse, the computer model placed their device either as first or second out of seven devices tested.

Conclusion: Adaptive or interactive conjoint analysis applied at the patient level can facilitate the choicemaking process whilst providing an insight into the relative importance of the key features that influence choice.

Medical decision making that is shared between staff and patients may result in greater satisfaction and adherence to therapy. ${ }^{1}$ The concept of shared decision making may be particularly challenging in the field of paediatrics where it is assumed that parents have overall responsibility for their children whilst the latter are expected to participate as much as possible when decisions are made about their future. ${ }^{2}$

Recombinant human growth hormone (rhGH) has been available for over 15 years for treatment of growth hormone $(\mathrm{GH})$ deficiency. ${ }^{3}$ However, daily, subcutaneous injection of rhGH is financially and physically costly and failure to comply with treatment may be as high as $50 \%$ of all cases. ${ }^{4}{ }^{5}$ To personalise therapy, the pharmaceutical industry has developed a number of different devices that vary in the method of subcutaneous injection, the injection product, the injection device and associated support services. ${ }^{6}$ However, the dramatic increase in the number of devices has made the overall process of providing unrestricted choice and shared decision making too time consuming in the clinic setting. In addition, there has been little progress in objectively identifying the factors that influence choice of device.

Conjoint analysis was originally developed for market research into consumer preferences, and is a method that investigates the relative importance of groups of attributes. ${ }^{7}$ More recently, it has been applied to various aspects of health care and has the potential to analyse patient preferences for various treatment alternatives. ${ }^{8-10}$

This paper reports on a novel application of conjoint analysis as a mechanism for facilitating choice of injection device in the outpatient clinic whilst improving our understanding of the factors that influence that choice.

\section{METHODS}

\section{Conjoint analysis methodology}

Conjoint analysis is a multivariate technique that is based on three interrelated concepts. ${ }^{11}$ Firstly, each product that is being assessed can be regarded as a bundle of potential "attributes"; secondly, each respondent has a unique value (or utility) for each attribute level, that is, each aspect of the product contributes towards the overall total value associated with the product; thirdly, combining these different utilities for the different attributes provides an individual's overall utility or preference for the specific product. To determine the utility values for each attribute, conjoint analysis uses a software programme that poses a series of very simple questions comparing two hypothetical products where each option is based on a different combination of attributes. The methodology has been used widely for understanding consumer preferences in aggregate, and is well validated in the literature. ${ }^{11} 12$

\section{Conjoint analysis model}

The values derived as above for each respondent can be added together to give the "value sum" associated with a defined device made up of several such attributes, still for that individual respondent, and that "value sum" can be compared with the "value sum" for other defined devices with different characteristics in order to model those of most appeal to the individual respondent. The conjoint analysis model developed for this application comprised 14 attributes related to device features and support services (table 1). Each attribute had between two and four utility levels. These attributes were identified as being important through previous discussions with patients, their parents and clinic staff. Some attributes (marked 
Table 1 Device attributes and utility levels included in the interview

\begin{tabular}{llll}
\hline Device attribute & Utility level & Device attribute & Utility level \\
\hline Bruising* & Almost none & Telephone helpline & $24 \mathrm{~h}$ a day \\
& A little & & from 9 am to $5.30 \mathrm{pm}$ \\
& Significant & None & Without a needle \\
Operated by & Button - automatic injection & Injection & With $0.5 \mathrm{~cm}$ long needle \\
& Pushing plunger (like a syringe) & & With $1 \mathrm{~cm}$ long needle \\
Pain felt* & Slight twinge & $9 \mathrm{~cm} \times 3 \mathrm{~cm}$ \\
& Distinct pinch & Size & $9 \mathrm{~cm} \times 6 \mathrm{~cm}$ \\
Weight & Like blood test & & $15 \mathrm{~cm} \times 6 \mathrm{~cm}$ \\
& Like a pencil $(10 \mathrm{~g})$ & $15 \mathrm{~cm} \times 9 \mathrm{~cm}$ \\
& Like half an apple (50 g) & & Available \\
& Like a standard apple $(100 \mathrm{~g})$ & Nurse support at home & Not available \\
Sound made & Like a large apple (150 g) & & Hidden/not used \\
& None & Needle visibility & A little visible \\
& Soft (heard by user only) & & Clearly visible \\
Dreparation & Distinct (heard within 2-3 m) & & In the fridge \\
Ease of holding ${ }^{*}$ & Loud (heard by all those in same room) & Stored & At room temperature \\
& No mixing required & & Delivered to home \\
& Mixing required weekly-to-monthly & Medicine & Available from local chemist
\end{tabular}

\footnotetext{
*Attributes marked with an asterisk were not included within the model and hence did not influence the choice based analysis.
}

with an asterisk in table 1) were deliberately not used in the calculations to model the respondents' device preferences as they are difficult to objectively quantify, but the data were nevertheless collected to assess their importance to the respondent. For all attributes, a higher level of utility indicates a greater preference for that aspect of the device.

\section{The on-screen interview}

Parents and children were interviewed by a specialist nurse using a computer-based interview that incorporated an interactive conjoint analysis module known as adaptive conjoint analysis (ACA, Sawtooth Software, Sequim, WA, USA). ${ }^{12}$ This computer-based interview was effectively a substitute for the routine discussion and demonstration of seven devices (table 2).
Such demonstrations previously occurred by face-to-face discussion as well as video demonstration with newly diagnosed $\mathrm{GH}$ deficient children, as well as those already on $\mathrm{rhGH}$ treatment. As the computer-based interview became a routine part of the clinic, the Yorkhill ethics committee approved the interview as part of clinical care. The parents generally found the interview understandable and had little or no trouble with task comprehension. After completion of the interview, the program used the responses of that individual to calculate the top three preferred devices and these were displayed on-screen. Further information was also available to the respondent which explained why the program had identified the three selected devices. The "Top 3 Identification" facility meant that the clinic staff, patient and parents did not have to consider all seven (or

Table 2 Specification of seven injection devices on the utility values of six attributes that were included in the conjoint model and which were rated the highest in the study

\begin{tabular}{|c|c|c|c|c|c|c|c|c|}
\hline \multirow[b]{2}{*}{ Attribute } & \multirow[b]{2}{*}{ Utility } & \multicolumn{7}{|c|}{ Injection device } \\
\hline & & A & B & C & D & $\mathbf{E}$ & $\mathbf{F}$ & G \\
\hline \multirow[t]{2}{*}{ Operated by } & Auto-injector & + & + & + & + & + & & + \\
\hline & Plunger & & & & & & + & \\
\hline \multirow[t]{4}{*}{ Weight } & Like a pencil $(10 \mathrm{~g})$ & & & & & & + & \\
\hline & Like half an apple $(50 \mathrm{~g})$ & & + & & + & + & & \\
\hline & Like a standard apple $(100 \mathrm{~g})$ & & & & & & & + \\
\hline & Like a large apple $(150 \mathrm{~g})$ & + & & + & & & & \\
\hline \multirow[t]{4}{*}{ Sound made } & None & & & & & & + & \\
\hline & Soft (heard by user only) & & & & + & + & & \\
\hline & Distinct (heard within $2 \mathrm{~m}$ ) & & + & & & & & + \\
\hline & Loud (easily heard by all those in same room) & + & & + & & & & \\
\hline \multirow[t]{2}{*}{ Preparation } & No mixing required & & & & + & & & + \\
\hline & Mixing required weekly-to-monthly & + & + & + & & + & + & \\
\hline \multirow[t]{3}{*}{ Needle } & Needle-free & + & & + & & & & + \\
\hline & $0.5 \mathrm{~cm}$ needle & & & & + & & & \\
\hline & $1 \mathrm{~cm}$ needle & & + & & & + & + & \\
\hline \multirow[t]{4}{*}{ Size } & $9 \mathrm{~cm} \times 3 \mathrm{~cm}$ & & & & & & + & \\
\hline & $9 \mathrm{~cm} \times 6 \mathrm{~cm}$ & & & & & & & + \\
\hline & $15 \mathrm{~cm} \times 6 \mathrm{~cm}$ & + & + & & + & + & & \\
\hline & $15 \mathrm{~cm} \times 9 \mathrm{~cm}$ & & & + & & & & \\
\hline
\end{tabular}


Would you prefer the device to the left or right? - select an appropriate level:

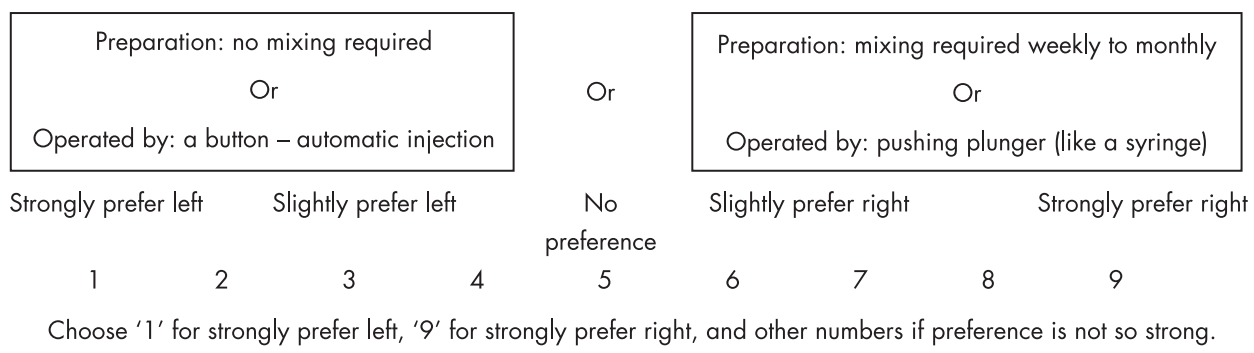

Figure 1 Adaptive conjoint: example screen from part of the interview.

more) devices on all aspects but would now be faced with a more manageable task relating to three devices only.

\section{Analysis of conjoint data}

The analyses involved in arriving at a personalised "Top 3" devices for each individual patient fell into two modules: one which generated the utility values and one which then calculated and displayed the respondent's "Top 3".

Module one: generation of utility values

At its core, conjoint requires the respondent to "trade-off" a series of alternative combinations of product features over several screens, similar to that shown in fig 1 . These combinations are selected by the program according to that same respondent's prior answers. As can be seen from fig 1, each respondent choice puts a relative weighting on each of the aspects shown on-screen, and after a series of such screens, in which different attributes are "traded-off", the program calculates the underlying utility values which "best fit" the answers given by this individual respondent. As the program cycles round, iteratively calculating values throughout the interview, it is able to display options that, more and more efficiently, arrive at an accurate representation of the respondent's unique value structure in the form of "utility values". Averaged across the entire set of respondents (or any subset), the aggregated mean utilities identify the motivations for choice of device (table 3). ${ }^{8-10}$ To allow easier interpretation of the data, these mean values have been recalculated such that the lowest level in any one attribute is set equal to zero, and results are presented as "differences" from that lowest level. The 3.38 for "bruising" in table 3 is the difference in utility between the two extreme levels of this attribute: thus, the larger the "value span" of a given attribute, the greater the defined "motivational value". To help in interpreting the relative appeal of each attribute and level, the attribute "bruising" (with the greatest value span of 3.38) was given an index of 100, and the remaining attributes scaled against this figure. Mathematically, this is justified as these values are only used for modelling in an "additive" context.

\section{Module two: calculation and display of top3}

Finally, the model was then immediately able to display the three most appropriate devices for subsequent consideration by the patient. This was achieved by the summation of the values derived for each specific respondent for each specific device aspect into "value sums", separately for each of seven existing available devices based on the values as shown in table 3 . The three most valued devices (ie, three highest "value sums" for that patient) were then automatically displayed on-screen.
Table 2 shows six of the actual elements that were summed to form the "value sums" of each existing device (A, B, C, D, E, F and $\mathrm{G}$ ).

\section{RESULTS}

\section{Subject characteristics}

Fifty six patients and their parents, attending a paediatric endocrine clinic at Yorkhill, participated in the interview. All families approached took part in this exercise. Out of these, 17 were already on rhGH treatment. The median (range) age of the patients was 13 years (3.5-24). In 11 out of 56 families, the interview was completed, solely, by the parents and in these cases the median age of the patient was 7 years (4.8-10). The interview took a median time of $18 \mathrm{~min}(12-30)$.

\section{Importance rating of attributes: aggregate results}

Lack of bruising was the highest rated attribute by the respondents and was designated an index of 100 (table 3). Compared to this, the other attributes in order of high to low desirability, in aggregate, were: auto-injector (98), lack of pain (93), lightweight (88), silent (82), ready-mixed (77), ease of holding (69), telephone helpline (66), needle-free (62), small size (60), nurse support (47), hidden needle (45), stored in fridge (13) and home delivery (6). It is clear that the most highly valued "device features" were automatic injection and weight, but that each of these may be outweighed by the more subjective aspects of likelihood of bruising and pain felt.

\section{Modelling choice of device: individual results}

Using the device specifications shown in table 2, "value sums" were calculated for seven devices and top three modelled. Device $D$ was calculated as the most popular followed by $G$ and $F$ (table 4). There were clear differences in the specifications of these devices and especially in the top six attributes (table 2). On comparison of the modelled results with the actual devices already being used by the 17 families with children on $\mathrm{rhGH}, 11$ had already been using devices that were regarded by the model as first or second preference (table 5). Only two had "suboptimum" devices, and of these one was modelled as a third choice and so clearly was still seen as meeting the patient's requirements. As noted above, the model was set up to select from a total of seven devices as possible candidates for the "Top 3 " so that the correlation is even more impressive than might appear from the above figures. Four out of 17 respondents who were on rhGH treatment were using device $\mathrm{E}$ and in three out of these four, device $\mathrm{D}$ was modelled as the first choice. The only differences between devices $\mathrm{E}$ and $\mathrm{D}$ are that the latter has a relatively shorter needle and its preparation does not require any mixing. 
Table 3 The mean utility values across the total sample for the 56 patients

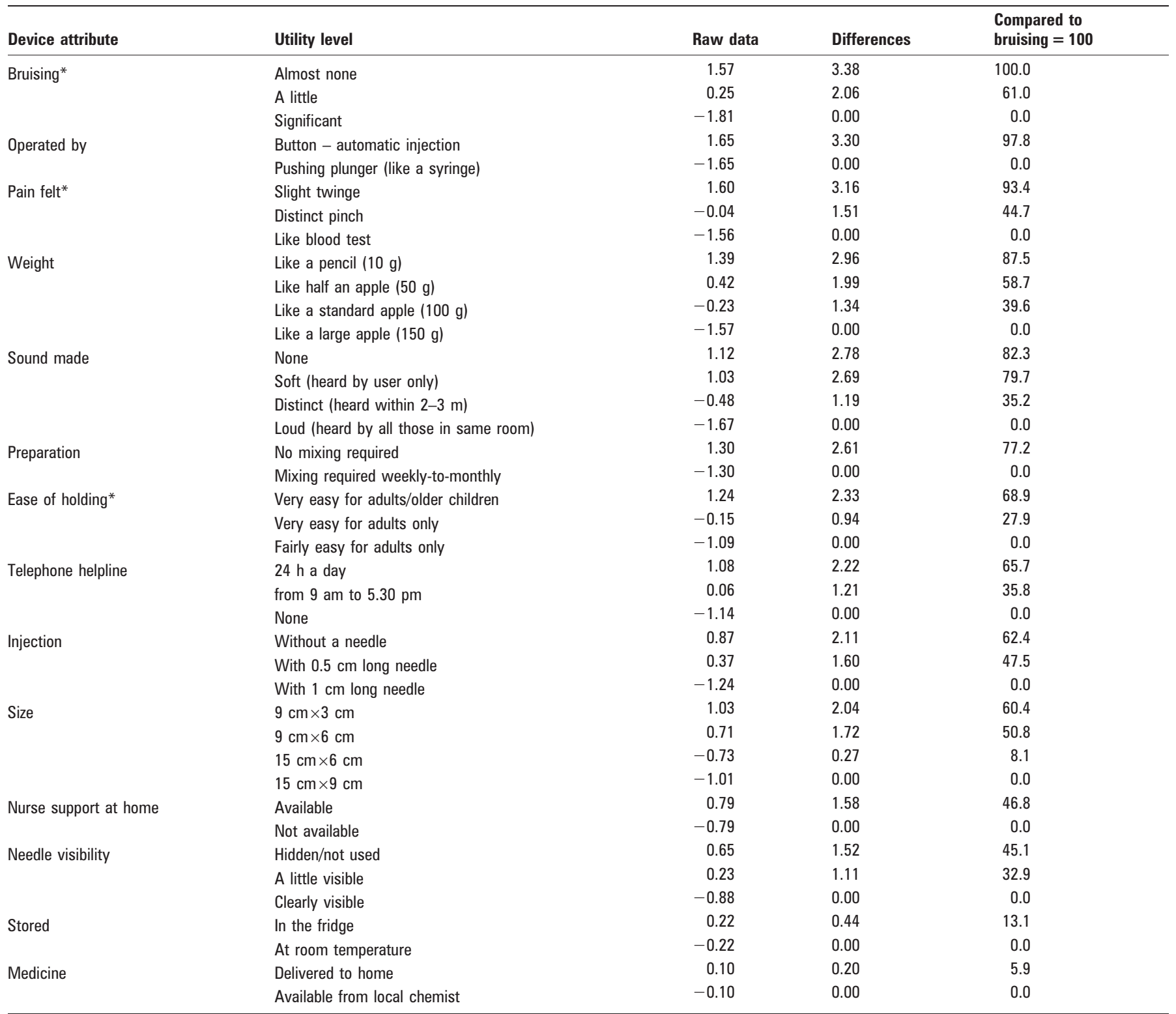

Attributes listed include those which were used to generate total device values and hence the "Top 3 " and those which were solely included for additional insight $\left({ }^{*}\right)$.

Modifying utility values of device attributes: aggregate results Since it is not solely the value of the different attributes that is recorded but the value of different levels of those attributes, conjoint analysis also provided data that judged the relative benefit of incrementally modifying attributes to improve their desirability. For example, the data from table 3 can be used to consider the relative merits of altering the level of three attributes and the subsequent change in relative patient appeal by modifying the device so that: (A) it is reduced in size from $15 \mathrm{~cm} \times 6 \mathrm{~cm}$ to $9 \mathrm{~cm} \times 3 \mathrm{~cm}$; (B) it changes from a $0.5 \mathrm{~cm}$ needle to a needle-free device; or (C) it becomes quiet in operation instead of creating a distinct sound. Alteration A leads to a change in utility values from 8.1 to 60.4 (value to patients $=52.3$ ), B leads to a change in utility values from 47.5 to 62.4 (value to patients $=14.9$ ), and $\mathrm{C}$ leads to a change in utility values from 35.2 to 82.3 (value to patients $=47.1$ ). Reducing the size of this particular device and making the injection noise-free are, therefore, more likely to increase patient acceptability than making the device needlefree.

\section{DISCUSSION}

This study has objectively investigated patient preferences for different attributes of delivery devices used for administering rhGH. It minimised any observer bias by using a self-completion methodology which, until now has rarely, if ever, been applied in this context. Not only has this study provided data that will facilitate the design and development of better and more patient-friendly devices, but we have also shown that this method of self-administered conjoint analysis-based choice can be readily adapted to the clinic setting and can generally be performed in less than $30 \mathrm{~min}$. Amongst the patients who were already on $\mathrm{rhGH}$ treatment, all the families had previously chosen their injection device following discussion with the clinic nursing staff. It was reassuring to observe that although there were some discrepancies, generally there was a clear correlation between the model projected devices and the actual devices used.

Our aggregated data clearly show that bruising and pain remain two of the greatest fears of patients and their parents and are strong motivators for device choice. We felt that we 
Table 4 The distribution of devices A-G amongst the "Top 3" modelled choices for device

\begin{tabular}{lccc}
\hline Device & $\begin{array}{l}\text { Modelled } \\
\text { choice 1 }\end{array}$ & $\begin{array}{l}\text { Modelled } \\
\text { choice 2 }\end{array}$ & $\begin{array}{l}\text { Modelled } \\
\text { choice 3 }\end{array}$ \\
\hline A & 0 & 2 & 6 \\
B & 5 & 6 & 8 \\
C & 3 & 5 & 7 \\
D & 23 & 18 & 12 \\
E & 0 & 5 & 6 \\
F & 11 & 9 & 6 \\
G & 14 & 11 & 11 \\
Total respondents & 56 & 56 & 56 \\
\hline
\end{tabular}

Table 5 Modelled derived preference of device against the actual device being used by the family in the 17 cases where a patient was receiving rhGH treatment

\begin{tabular}{llllll}
\hline Injection device & B & C & D & E & F \\
\hline $\begin{array}{l}\text { Number of families using device } \\
\text { Modelled derived preference }\end{array}$ & 1 & 2 & 9 & 4 & 1 \\
$\quad$ First & & 2 & 6 & & \\
$\quad$ Second & 1 & & 2 & & \\
$\quad \begin{array}{l}\text { Third } \\
\text { Lower than third }\end{array}$ & & & & & 1 \\
\hline
\end{tabular}

could not specify the utility values for these attributes for the devices included in the study as these attributes are dependent greatly on perception. However, it is clear that when considering device design, manufacturers would benefit from features that would be perceived as causing the least amount of bruising and pain (ie, look unthreatening), even if this may be at the expense of more tangible but less highly regarded features. Clinical staff, like manufacturers, can also draw useful conclusions, for example that reassurance and training to minimise pain are obvious priorities, but operation, weight and sound, as defined, are all aspects with almost as much value to the patient.

Furthermore, the data obtained from this report can also be used to weigh the resources required in any device alteration against the gain in "patient appeal". For instance, it is likely that the expertise and the expense involved in making a device sound-free is greater than reducing the size of the device, thus encouraging the manufacturer towards concentrating on the second alteration. Similar inferences can be drawn regarding device specification changes that involve combining improvements; since the utility values calculated can be added together, the total increase in utility of two or more simultaneous changes can be identified.

There is scarce information on how patients view community-based support services, yet these may be resource intensive for many pharmaceutical companies. Our data suggest that, whilst the availability of a telephone helpline was rated quite highly, the need for nurse support at home was much less highly valued. Home delivery of drugs, a service often hailed by firms, was rated as of least importance. It is, of course, possible that the need for some of these additional support features may be influenced by geographical and socioeconomic factors.

To some extent, certain attributes may be correlated with each other, such as automatic injection and noise, needle-free

\section{What is already known on this topic}

- Children and their parents are often encouraged to make a choice of treatment options.

- Objectively identifying factors that influences choice is often difficult.

\section{What this study adds}

- A short interview using conjoint analysis as an interactive programme facilitates choice.

- Conjoint analysis provides information that can improve the future design of injection devices.

and noise, or weight and size. Therefore, some care is needed in mathematically applying all of these values to a device, since ideally all attributes are orthogonal. The test-retest reliability of the model was not assessed as it was felt that the response at the second interview may be biased and that the interview process would have been too demanding for most families attending the outpatient clinic. A previous study has found a high degree of reproducibility within a 2-month period. ${ }^{7}$

In summary, we describe a short, objective method of providing a personalised "short list" from the wide range of rhGH injection devices available, via a method that also provides helpful information to guide the future development of devices.

Acknowledgements: The authors thank Rafaat Rahmani for initial discussion and direction and Paul Finer for programming the total interview and algorithms for "Top 3" display, around the Sawtooth module. WS was supported by funding from Proctor \& Gamble, Ferring and Serono UK whilst working on this project.

Competing interests: SFA has received funding towards research and development from Aventis, Ferring, Novo Nordisk, Pfizer, Proctor \& Gamble and Serono UK and has served on the advisory boards of Ferring, Novo Nordisk and Serono UK.

\section{REFERENCES}

1. Speedling EJ, Rose DN. Building an effective doctor-patient relationship: from patient satisfaction to patient participation. Soc Sci Med 1985;17:115-20.

2. Children Act 2004. Chapter 31.Norwich, UK: Stationary Office, 2005.

3. Hintz RL. Growth hormone: uses and abuses. BMJ 2004;328:907-8.

4. Hindmarsh PC, Brook CG. Compliance with growth hormone treatment - is it a problem? Horm Res 1999;51(Suppl 3):104-8.

5. Wetterau L, Cohen P. New paradigms for growth hormone therapy in children. Horm Res 2000;53(Suppl 3):31-6.

6. Fidotti E. A history of growth hormone injection devices. J Pediatr Endocrinol Metab 2001;14:497-501.

7. Ryan M, Scott DA, Reeves C, et al. Eliciting public preferences for healthcare: a systematic review of techniques. Health Technol Assess 2001;5:1-186.

8. Johansson G, Stallberg B, Tornling G, et al. Asthma treatment preference study: a conjoint analysis of preferred drug treatments. Chest 2004;125:916-23.

9. Zimet GD, Mays RM, Sturm LA, et al. Parental attitudes about sexually transmitted infection vaccination for their adolescent children. Arch Pediatr Adolesc Med 2005:159:132-7.

10. Fraenkel L, Constantinescu F, Oberto-Medina M, et al. Women's preferences for prevention of bone loss. J Rheumatol 2005;32:1086-92.

11. Luce D, Tukey J. Simultaneous conjoint measurement: a new type of fundamental measurement. J Math Psychol 1964;1:1-27.

12. Orme BK. Getting started with conjoint analysis. Madison, Wl: Research Publishers, 2006. 